\title{
Análise da Quantidade de Sementes Necessárias para a Segmentação Semi-Automática em Imagens de TC de Pulmão Utilizando Crescimento de Região
}

\author{
Glenda Proença Train ${ }^{1}$, Jeovane Honório Alves ${ }^{1}$, Lucas Ferrari de Oliveira ${ }^{1}$ \\ ${ }^{1}$ Universidade Federal do Paraná \\ Curitiba - PR, Brasil \\ \{gpt16, jhalves, lferrari\}@inf.ufpr.br
}

\begin{abstract}
Among all cancer types, lung cancer is the third one with most incidences and it holds the highest mortality rate worldwide. Lung diseases such as cancer and ILDs (Interstitial Lung Diseases) have a greater chance of cure when diagnosed early. Aiming to contribute to the realization of these diagnoses, this research proposes a semi-automatic method of lung segmentation based on the technique of region growing and presents an impact analysis of the position and quantity of used seeds. Several experiments were performed on two datasets, HUG-ILD and Vessel 12, resulting in Dice coefficients of $96,94 \% \pm 1,41 \%$ and $96,83 \% \pm 0,92 \%$, respectively.
\end{abstract}

Resumo. Dentre as categorias de câncer, o câncer de pulmão é o terceiro mais incidente e detém a maior taxa de mortalidade mundial. Doenças pulmonares, como câncer e ILDs (Interstitial Lung Disease), possuem uma maior possibilidade de cura quando diagnosticadas precocemente. Visando contribuir para a realização desses diagnósticos, essa pesquisa propõe um método semi-automático de segmentação de pulmão baseado na técnica de crescimento de região e apresenta uma análise do impacto da localização e quantidade das sementes utilizadas. Diversos experimentos foram realizados em duas bases, HUG-ILD e Vessel 12, obtendo coeficientes Dice de 96,94\% \pm $1,41 \%$ e $96,83 \% \pm 0,92 \%$, respectivamente.

\section{Introdução}

De acordo com a IARC (International Agency for Research on Cancer), o câncer de pulmão ocupa a terceira posição entre as diferentes categorias de câncer com maior incidência. Mas em questão de mortalidade, o câncer de pulmão possui os maiores índices mundiais [IARC 2018]. Segundo os dados do INCA (Instituto Nacional de Câncer), no Brasil, apenas $16 \%$ dos casos de câncer são diagnosticados em estágio inicial [INCA 2020]. Além do câncer, as ILDs (Interstitial Lung Diseases), que abrangem cerca de 200 diferentes tipos de problemas pulmonares, também podem danificar os tecidos dos pulmões resultando em uma expectativa de vida de dois ou três anos [Thoracic 2020].

Visando auxiliar e acelerar o diagnóstico, os sistemas CAD (Computer-Aided Diagnosis) têm uma grande importância para aumentar a chance de sobrevivência dos pacientes. Geralmente, os sistemas CAD para diagnóstico de câncer de pulmão são 
compostos por quatro etapas: segmentação dos pulmões, detecção dos nódulos pertencentes aos pulmões, segmentação dos nódulos detectados e diagnóstico dos nódulos em maligno e benigno [Vannier et al. 2013].

Nos últimos anos, diversos métodos foram desenvolvidos para segmentar as áreas pulmonares visando contribuir para a primeira etapa dos sistemas CAD. Entre os trabalhos mais recentes, foi proposta uma abordagem adaptativa que combina técnicas como Linear Combination of Discrete Gaussian (LCDG) e Markov-Gibbs Random Fields (MGRF), chegando à um coeficiente Dice de $99.0 \% \pm 0.5 \%$ no conjunto de imagens Vessel 12 [Soliman et al. 2017].

Além das abordagens probabilísticas, os modelos de deep learning se tornaram frequentes recentemente. Em [Harrison et al. 2017] é proposta uma rede neural convolucional totalmente conectada, chamada de P-HNN (Progressive Holistically-Nested Network), a qual tem o objetivo de aprimorar a segmentação de modo que a rede consiga lidar com variações de aparência sem afetar seu desempenho em variações de forma, obtendo um resultado de 97,9\% $\pm 1,0 \%$ na base de imagens HUG-ILD. Por fim, em [Alves et al. 2018], é proposta uma abordagem utilizando uma rede neural convolucional, e esse modelo é complementado pela técnica Conditional Random Fields (CRF) que analisa a vizinhança e aumenta a quantidade de pixels verdadeiros positivos, refinando a segmentação. Essa combinação de modelos resultou em um coeficiente Dice de $98,67 \% \pm 0,94 \%$ e de $99,19 \% \pm 0,37 \%$ nas bases HUG-ILD e Vessel 12, respectivamente.

Este trabalho propõe um método semi-automático de segmentação da região pulmonar utilizando técnicas de crescimento de região e morfologia matemática, além de criar novas abordagens para encontrar parâmetros, tratar irregularidades e recuperar fragmentos perdidos, pertencentes à região de interesse, ao usar as técnicas mencionadas. Ademais, é realizada uma análise da influência da quantidade e localização dos pontos sementes utilizados na etapa de crescimento de região.

\section{Bases de imagens}

O método proposto se baseou em dois conjuntos de imagens diferentes, HUG-ILD e Vessel 12, que serão descritos nesta seção. Ambas as bases possuem fatias de 512x512 pixels.

\subsection{HUG-ILD}

Elaborada pelos hospitais universitários de Geneva (HUG - University Hospitals of Geneva), essa coleção de imagens abrange casos de doenças pulmonares intersticiais (ILDs - Interstitial Lung Diseases), que podem ser descritas como alterações graduais nos pulmões, podendo levar à disfunção respiratória [Depeursinge et al. 2012]. A escolha destas imagens foi baseada na variabilidade e dificuldade de realizar o processo de segmentação em exames que apresentam ILDs.

A base contém imagens dos exames de tomografia de 128 pacientes diagnosticados com diferentes tipos de ILDs. Cada exame possui em média 25 fatias, sendo suas máscaras binárias. Somente 118 exames foram utilizados nesta pesquisa devido à existência de máscaras incompletas. Exemplo da base na Figura 1, à esquerda. 


\subsection{Vessel 12}

Proposta em 2012 para comparar o desempenho de métodos automáticos e semi-automáticos de identificação de vasos em tomografias computadorizadas da caixa torácica, a Vessel 12 (VESsel SEgmentation in the Lung) disponibiliza um total de 23 exames (20 para teste e 3 exemplos), com média de 430 fatias por exame [Rudyanto et al. 2014]. Exemplo da base na Figura 1, à direita, abaixo.
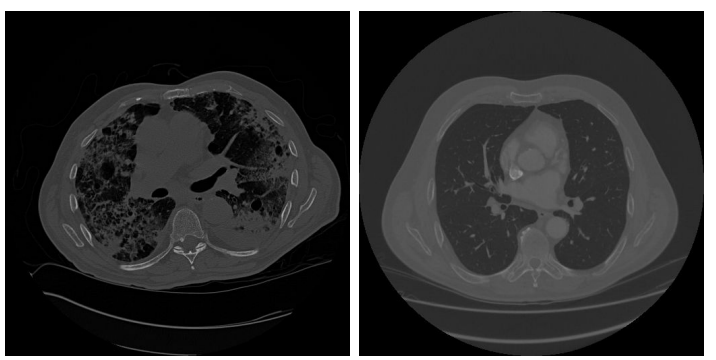

Figura 1. Exemplos de imagem da base HUG-ILD, à esquerda, e Vessel 12, à direita.

\section{Metodologia}

Ambos os conjuntos de exames, mencionados na seção anterior, fornecem imagens no formato DICOM (Digital Imaging and Communications in Medicine). Inicialmente, as imagens foram lidas na escala Hounsfield (HU) e depois convertidas para a escala de cinza com transformação linear.

A Figura 2 exemplifica as etapas da metodologia, iniciando com o crescimento de região (a), aplicação do preenchimento (b), uso do fechamento (c), suavização das bordas com blocos menores (d) e maiores (e), aplicação da técnica de erosão (f), uso de técnicas de refinamento das bordas de acordo com as linhas (g) e colunas (h) e, por fim, separação dos pulmões (i). As descrições de cada uma das etapas serão apresentadas nesta seção.

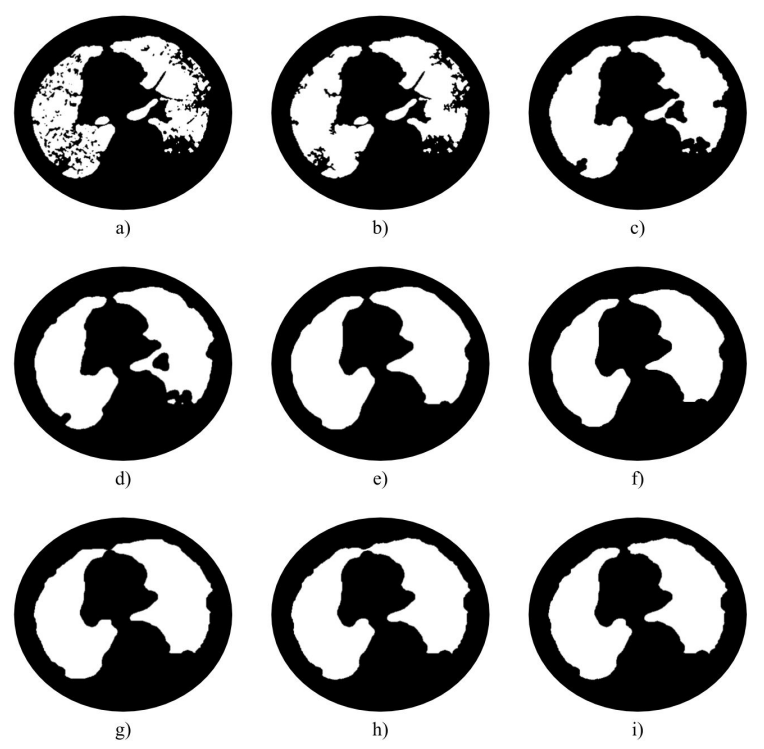

Figura 2. Etapas da metodologia proposta para segmentação de pulmões. 


\subsection{Escolha das sementes}

Uma das principais etapas da metodologia proposta foi o uso do algoritmo de crescimento de região, que adicionava à segmentação os pixels conectados a uma coordenada inicial, nas três dimensões (x, y, z), e que estavam dentro de um determinado intervalo. Assim, esse algoritmo contava com a ajuda de uma lista de coordenadas pertencentes aos pulmões para que fosse possível separar a região de interesse das outras estruturas. Dessa forma, havia a necessidade do conhecimento de um especialista para marcar determinadas áreas das imagens (sementes), garantindo que essas coordenadas pertencessem aos pulmões.

Para encontrar essas sementes, três formas semi-automáticas foram propostas. Essas modalidades são apresentadas na Tabela $1 \mathrm{e}$ foram denominadas de center, edge e random.

Tabela 1. Categorias de localidade das sementes inseridas.

\begin{tabular}{|c|l|l|}
\hline Categoria & \multicolumn{1}{|c|}{ Objetivo } & \multicolumn{1}{|c|}{ Descrição } \\
\hline Center & $\begin{array}{l}\text { Em cada região do pulmão, } \\
\text { encontrar uma semente próxima } \\
\text { à área central do mesmo. }\end{array}$ & $\begin{array}{l}\text { Para cada região de interesse, na imagem da } \\
\text { máscara na fatia central (eixo z), ocorrem três } \\
\text { esões (kernel de elipse de 5x5) e o centróide } \\
\text { do resultado é calculado e usado como } \\
\text { semente. }\end{array}$ \\
\hline Edge & $\begin{array}{l}\text { Em cada pulmão, encontrar uma } \\
\text { semente próxima à região da da } \\
\text { borda pulmonar. }\end{array}$ & $\begin{array}{l}\text { Semelhante ao center, mas o centróide é } \\
\text { calculado na subtração da fatia central (eixo z) } \\
\text { da máscara menos a imagem obtida nas } \\
\text { erosões. }\end{array}$ \\
\hline Random & $\begin{array}{l}\text { Em cada pulmão, encontrar uma } \\
\text { semente em uma localidade } \\
\text { aleatória. }\end{array}$ & $\begin{array}{l}\text { Para cada pulmão, na imagem da máscara na } \\
\text { fatia central (eixo z), é gerada uma } \\
\text { coordenada (x, y) aleatória. Essa coordenada é } \\
\text { usada como semente. }\end{array}$ \\
\hline
\end{tabular}

Após determinar as sementes nos pulmões, é necessário validar suas coordenadas. Para isso, existe um teste de validação. $\mathrm{O}$ teste tem como princípio verificar se as coordenadas estão dentro da máscara disponibilizada pela base.

Teste de validação: verifica se as coordenadas $(x, y)$ encontradas resultam em um pixel com valor igual a 255 nas imagens da máscara. Especificamente, nas modalidades center e random, esse teste é feito na fatia central, nas três fatias anteriores e nas três posteriores. Já na modalidade edge, somente a fatia central é testada. Se o teste é verdadeiro, as coordenadas são consideradas válidas e inseridas na lista de sementes requisitada pelo algoritmo de crescimento de região. Caso as coordenadas não passem no teste, é utilizado um algoritmo de movimentação, que será apresentado a seguir.

Movimentação de uma semente inválida: caso a primeira tentativa de encontrar uma semente resulte em uma coordenada inválida, é necessário encontrar novas coordenadas e realizar novamente o teste de validação. Assim, a imagem da máscara em que a semente inválida se encontra é dividida em quadrantes e, de acordo 
com cada quadrante, é definida uma prioridade de movimentação, direcionada para o centro do pulmão. Essa movimentação é feita pixel a pixel e o teste de validação é realizado a cada movimentação.

\subsection{Aumento das sementes}

Além de investigar qual o impacto da localização da semente, essa pesquisa também tem o objetivo de descobrir a influência do número de sementes ao segmentar um pulmão. Dessa forma, foram desenvolvidas 3 categorias diferentes para controlar o número de sementes, chamadas de single, aux e radius, que são apresentadas na Tabela 2.

Tabela 2. Categorias de aumento da quantidade de sementes.

\begin{tabular}{|c|l|l|}
\hline Categoria & \multicolumn{1}{|c|}{ Objetivo } & \multicolumn{1}{c|}{ Descrição } \\
\hline Single & Utilizar poucas sementes. & $\begin{array}{l}\text { Somente as sementes encontradas pelos métodos } \\
\text { anteriores são usadas. }\end{array}$ \\
\hline Aux & $\begin{array}{l}\text { Considerar a influência do do } \\
\text { volume na escolha das } \\
\text { sementes. }\end{array}$ & $\begin{array}{l}\text { Para cada uma das sementes encontradas são geradas } \\
\text { novas sementes, usando as mesmas coordenadas (x, } \\
\text { y), mas alterando a fatia do pulmão (eixo z). As } \\
\text { fatias que foram utilizadas nesse processo são as três } \\
\text { fatias anteriores e as três posteriores à fatia central. }\end{array}$ \\
\hline Radius & $\begin{array}{l}\text { Considerar as coordenadas } \\
\text { em áreas próximas às } \\
\text { sementes já escolhidas. }\end{array}$ & $\begin{array}{l}\text { Para cada uma das sementes encontradas são } \\
\text { selecionadas novas sementes dentro de um raio, } \\
\text { chamado de shift. O shift é a distância dos 8 vizinhos } \\
\text { da semente para a própria semente. As sementes } \\
\text { selecionadas podem ser aquelas pertencentes ao raio } \\
\text { ou aquelas entre a semente e o raio, como é mostrado } \\
\text { na Figura 3, onde flood é a variável que faz o } \\
\text { controle das sementes selecionadas. }\end{array}$ \\
\hline
\end{tabular}
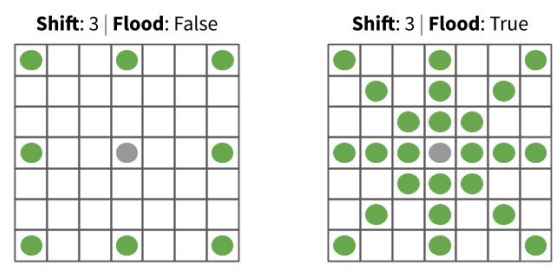

Figura 3. Exemplo do controle de inserção de sementes na categoria radius.

\subsection{Busca dos parâmetros do crescimento de região}

Como já mencionado, a técnica utilizada foi o crescimento de região, baseado em threshold. Além das sementes, essa técnica necessitava de dois parâmetros denominados lower e upper. Essencialmente, o algoritmo substitui os pixels dentro do intervalo entre lower e upper por um determinado valor, geralmente 255, se baseando na localização das sementes. O problema encontrado ao utilizar esta técnica foi que esses parâmetros variavam muito de uma imagem para outra, até mesmo para as 
imagens de exames da mesma base de dados. Sendo assim, buscamos criar uma forma de encontrar tais parâmetros automaticamente.

O primeiro passo para a solução foi separar os exames de imagens mais claras dos exames com imagens mais escuras, pois essa característica das imagens afetava consideravelmente os valores dos parâmetros. Para isso, calculamos a média da equalização por histograma de todas as imagens do exame. Se essa média resultasse em um valor maior do que 100, as imagens do exame seriam consideradas claras, caso contrário, seriam consideradas normais.

Na próxima etapa, realizamos uma Grid Search, que testou uma determinada quantidade de valores de parâmetros, tanto lower quanto upper, escolhidos previamente. Para o caso em que as imagens do exame foram consideradas normais, o lower variava entre 0 e 15 e o upper entre 25 e 75 , ambos aumentando de uma unidade a cada iteração. Quando as imagens foram consideradas claras, o lower aumentava de 0 a $110 \mathrm{e}$ o upper de 50 a 140, variando uma unidade e 10 unidades, respectivamente.

Para cada iteração da Grid Search, o algoritmo de crescimento de região foi executado com as sementes encontradas previamente e os parâmetros atuais do laço. Após essa segmentação prévia, os números de pixels pretos e brancos do volume foram contabilizados. Com esses valores, calculamos uma proporção do número de pixels brancos sobre o número de pixels pretos. Essa proporção funcionou como uma função de custo pois, se ela estivesse dentro de um certo limite, os valores atuais de lower e upper seriam considerados como candidatos e passariam por uma verificação para serem escolhidos ou descartados. O limite escolhido foi entre 3,9\% e 35\%.

Essa verificação em que os candidatos foram submetidos se baseava em um sistema de votação. Um laço foi executado, variando do valor do upper candidato menos cinco unidades até o upper mais cinco unidades. Em cada iteração, o mesmo teste de proporção foi realizado e, se a proporção estivesse dentro do limite, um voto seria contabilizado. Além disso, o aparecimento, ou não, de uma proporção média foi considerado caso o valor fosse menor que $20 \%$. Ao final do laço, se todas as iterações tivessem recebido um voto (totalizando 11 votos) e, entre elas, uma proporção média tivesse aparecido, a verificação indicava que um bom par de parâmetros havia sido encontrado, caso contrário, informava que outro par deveria ser buscado.

A principal ideia da verificação foi encontrar um par de parâmetros que não ignorasse muitas áreas pertencentes ao pulmão e nem considerasse outras estruturas como sendo parte do pulmão. Assim, um bom upper foi aquele que, ao mudar um pouco do seu valor, não alterasse tanto a proporção. Como o lower era geralmente 0 e, para valores maiores ele era bem sensível, não foi necessário modificá-lo dentro dessa verificação.

Após encontrar um bom par de parâmetros, havia um último laço que aumentava os parâmetros de uma em uma unidade e realizava novamente a verificação. Esse laço continuava enquanto a verificação indicasse que os parâmetros eram bons. Essa última etapa se justifica pelo motivo de que há a possibilidade de algumas partes dos pulmões estarem sendo perdidas caso só apareçam proporções mais baixas, próximas a 3,9\%. Ao fim das iterações, os últimos valores válidos de lower e upper se tornavam os parâmetros que seriam usados na segmentação. 
O tempo de processamento dessa etapa, em uma arquitetura Intel ${ }^{\circledR}$ Core $^{\mathrm{TM}}$ i9-9900K, com 32K de L1, 256K de L2 e 16384K de L3, 8 núcleos e 65803696 KB de memória, foi, em média, 15 segundos por exame na base HUG-ILD e 7 minutos por exame na Vessel 12.

\subsection{Aplicação de técnicas de morfologia matemática}

Após encontrar todos os parâmetros necessários para o crescimento de região, as etapas do processo de segmentação se iniciavam. O primeiro passo foi aplicar um filtro de mediana sobre as imagens do exame, com um kernel de tamanho $3 \times 3$. Esse passo removeu pequenos ruídos que poderiam existir nas imagens. O segundo passo foi a aplicação do algoritmo de crescimento de região, baseado em threshold, com as sementes e os valores de lower e upper. Nesse momento, o exame já tinha uma segmentação inicial, mas ela continha muitos buracos e irregularidades, como é possível observar na Figura 2a). Para resolver esses problemas, foi aplicado um preenchimento seguido de um fechamento, com uma elipse de tamanho $11 \times 11$. O preenchimento ajudou a fechar os buracos internos do pulmão, Figura $2 \mathrm{~b}$ ), enquanto que o fechamento serviu para tratar as quebras e irregularidades das bordas do pulmão, Figura 2c).

\subsection{Suavização das bordas}

Mesmo com a aplicação das técnicas de morfologia matemática, a segmentação ainda estava longe do ideal, pois as bordas dos pulmões estavam fragmentadas e, após o fechamento, novos buracos surgiram dentro da área segmentada. Assim, com o objetivo de mitigar essas falhas, foi criada uma técnica chamada de suavização. Essa técnica consistiu em quebrar a imagem segmentada em vários blocos pequenos de tamanho fixo, aplicar o fecho convexo em cada um desses blocos e reconstruir a imagem. Aplicando esse método em todo o volume do exame, os buracos foram cobertos e as bordas dos pulmões se tornaram uniformes, como é possível observar na Figura 4.

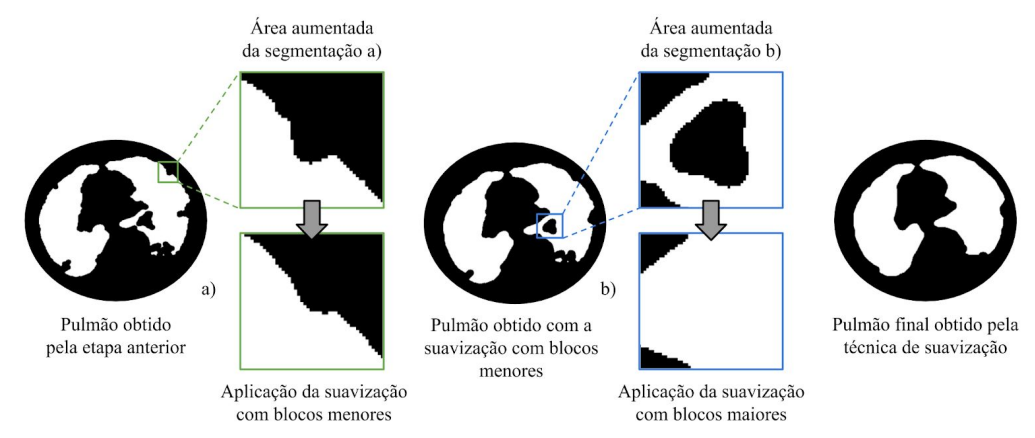

Figura 4. Exemplo de aplicação da técnica de suavização.

Essa técnica foi aplicada duas vezes, sendo que na primeira vez houve uma suavização com blocos menores, de tamanho 16x16, com o objetivo de complementar as bordas sem deformá-las, Figura 2d). Já na segunda aplicação os blocos eram maiores, de tamanho 23x23, e essa etapa tinha a intenção de cobrir as falhas maiores que se encontravam dentro dos pulmões, Figura 2e). Uma erosão, com kernel de elipse de tamanho 11x11, foi aplicada logo após a segunda suavização com o propósito de afinar levemente as bordas aumentadas pela suavização. O resultado da erosão é apresentado na Figura 2f). 


\subsection{Refinamento das bordas}

Após a execução da etapa de suavização foi possível notar que algumas partes das bordas não correspondiam ao contorno pulmonar ou estavam sendo perdidas. Com o objetivo de recuperar essas partes perdidas, foi proposto um método chamado refinamento. A essência do refinamento se baseou na ideia de um bit de paridade que indicava quando se estava dentro ou fora do pulmão.

A imagem segmentada foi percorrida, linha a linha e, quando um pixel pertencente ao pulmão era encontrado, o bit de paridade recebia um valor verdadeiro. Como a intenção era recuperar as bordas, ao invés de continuar percorrendo a imagem da esquerda para a direita, o algoritmo começava a percorrer a imagem na direção oposta, avaliando os pixels que poderiam ser candidatos à pertencerem ao pulmão. Cada pixel candidato passou por um teste de limiar para que houvesse uma decisão se seria incluído, ou não, na área segmentada. Esse teste é mostrado na equação (1), onde média é a média da área segmentada da imagem da fatia $s$, desvio ó o desvio padrão da área segmentada da imagem da fatia $s$ e $c$ é uma constante igual a 1 em imagens claras (caso em que a média dos valores da imagem da fatia $s$ é maior que 70) e 2 em imagens normais.

$$
\text { média }_{s}-\text { c.desvio } \leq \text { valor pixel } \leq \text { média }_{s}+\text { c.desvio }
$$

Assim que a busca pela área pulmonar terminasse (teste do limiar falso) a próxima borda do pulmão era buscada e o processo se repetia. Além de realizar o refinamento linha a linha, a técnica foi aplicada coluna a coluna, como é apresentado pela Figura 2g) e Figura 2h), respectivamente.

A Figura 5 exemplifica o efeito do algoritmo nas bordas dos pulmões, iniciando com o pulmão obtido após a suavização (a), com a área aumentada desse pulmão que passará pelo refinamento linha a linha (b), resultado obtido nessa área após o refinamento linha a linha (c), pulmão obtido depois do refinamento linha a linha (d), área aumentada que irá passar pelo refinamento coluna a coluna (e), resultado obtido nessa área após o refinamento coluna a coluna (f) e, por fim, o pulmão obtido após todo o processo de refinamento $(\mathrm{g})$.

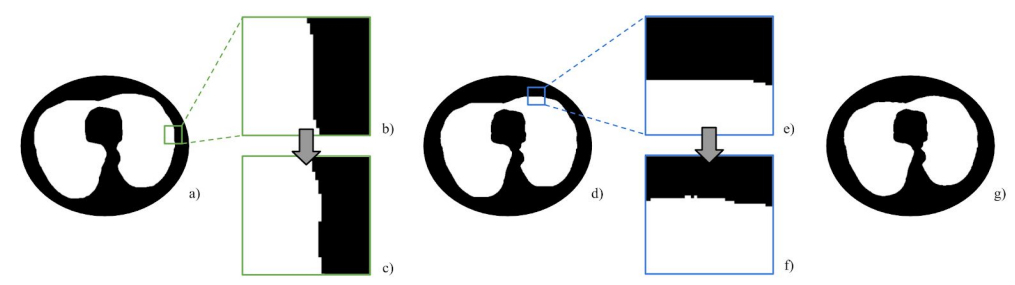

Figura 5. Exemplo de aplicação da técnica de refinamento.

\subsection{Separação dos Pulmões}

Nas etapas anteriores, algumas áreas dos pulmões ficavam unidas, aumentando o número de falsos positivos. Dessa forma, foi proposta uma solução de três etapas para separar os pulmões.

Inicialmente, foi necessário escolher quais imagens deveriam passar por esse processo, pois em grande parte do volume do exame os lobos dos pulmões estavam 
separados, assim, aplicar desnecessariamente esse método só consumiria tempo de processamento. As imagens que possuíam apenas um componente segmentado foram selecionadas. Dentre essas imagens, passariam pelo método de separação dos pulmões àquelas que tivessem um tamanho maior do que a área média das fatias.

O próximo passo consistiu em encontrar a região em que havia uma maior possibilidade de estar localizada a junção dos dois pulmões. Para isso, os componentes únicos, encontrados anteriormente, passaram por várias erosões até serem separados em dois pedaços com tamanho de contorno maior que 50 (pedaços menores eram descartados). Com esses dois componentes, os centróides deles foram calculados e o ponto médio encontrado. Supondo que o ponto estava em $\left(x_{m}, y_{m}\right)$, uma área de interesse foi gerada em volta desse ponto, com $x$ iniciando em $x_{m}-30$ e indo até $x_{m}+30$ e com $y$ iniciando em 0 e indo até 512 (número de linhas da imagem).

Por fim, foi realizado o último passo em que a separação dessas áreas foi efetuada. Dispondo da área de interesse, a sua média, $m$, e o seu desvio padrão, $d$, foram calculados e dois limites foram definidos, um inferior, sendo $m-2 * d$, e um superior, sendo $m+2 * d$. Após isso, todas as posições da área de interesse foram percorridas na imagem segmentada e, se o pixel tivesse um valor menor do que o limite inferior ou tivesse um valor maior que o limite superior ele era removido da imagem segmentada, ou seja, recebia o valor 0. Para suavizar as áreas modificadas da imagem foram aplicadas duas dilatações seguidas de duas erosões com kernel de tamanho 5x5 com o formato de uma elipse. $\mathrm{O}$ resultado pode ser visto na Figura $2 \mathrm{i}$ ).

\section{Experimentos}

Os mesmos testes foram realizados nas bases de imagens, HUG-ILD e Vessel 12, e para cada uma das modalidades de escolha de sementes (center, edge, random). Dessa forma, foram realizados um total de 12 experimentos diferentes. As descrições são mostradas na Tabela 3 abaixo. Para determinar o tamanho dos kernels, mencionados anteriormente, foram testados kernels quadrados, de tamanho $2 \times 2$ à $25 \times 25$, e selecionados aqueles que apresentaram melhores resultados ao realizar todo o processo de segmentação.

Tabela 3. Descrição dos experimentos realizados e quantidade de sementes utilizadas em cada um deles.

\begin{tabular}{|c|l|c|}
\hline Exp. & \multicolumn{1}{|c|}{ Descrição } & $\begin{array}{c}\text { N. }{ }^{\text {o de }} \\
\text { Sementes }\end{array}$ \\
\hline 1 & $\begin{array}{l}\text { Somente as duas sementes, encontradas inicialmente na escolha de } \\
\text { sementes, foram utilizadas. Esse experimento usou poucas sementes para } \\
\text { descobrir a relevância delas na metodologia. }\end{array}$ & 2 \\
\hline 2 & $\begin{array}{l}\text { Além das duas sementes, encontradas inicialmente, outras sementes foram } \\
\text { geradas usando o método aux. Esse teste teve o objetivo de avaliar o } \\
\text { impacto do aumento de sementes usando o volume. }\end{array}$ & 14 \\
\hline 3 & $\begin{array}{l}\text { Além das duas sementes, encontradas inicialmente, o método radius foi } \\
\text { utilizado à 5 pixels de distância com a variável flood sendo falsa. O impacto } \\
\text { do aumento de sementes usando a proximidade foi o objetivo deste teste. }\end{array}$ & 18 \\
\hline 4 & Além das duas sementes, encontradas inicialmente, o método radius foi & 322 \\
\hline
\end{tabular}




\begin{tabular}{|l|l|l|}
\hline & $\begin{array}{l}\text { utilizado à } 20 \text { pixels de distância com a variável flood sendo verdadeira. } \\
\text { Esse teste visou avaliar o impacto do aumento excessivo de sementes } \\
\text { usando a proximidade. }\end{array}$ & \\
\hline
\end{tabular}

Os resultados das segmentações foram avaliados com o coeficiente de similaridade Dice (SDC - Dice Similarity Coefficient), que avalia a sobreposição entre a segmentação obtida e a respectiva máscara [Zou et al. 2004]. Sabendo o número de verdadeiros positivos (VP), verdadeiros negativos (VN), falsos positivos (FP) e falsos negativos (FN), o coeficiente pode ser calculado pela equação (2):

$$
S D C=\frac{2 V P}{2 V P+F P+F N}
$$

Para resumir as informações, serão apresentados somente os resultados de média e desvio padrão dos experimentos.

\section{Resultados}

Na Tabela 4 são apresentados os resultados obtidos na base de imagens HUG-ILD e Vessel 12.

Tabela 4. Coeficientes Dice médios obtidos nos experimentos realizados nas bases HUG-ILD e Vessel 12.

\begin{tabular}{|c|c|c|c|c|c|c|}
\hline & \multicolumn{3}{|c|}{ Base HUG-ILD } & \multicolumn{3}{c|}{ Base Vessel12 } \\
\hline Exp. & Center $(\%)$ & Edge $(\%)$ & Random $(\%)$ & Center $(\%)$ & Edge (\%) & Random (\%) \\
\hline 1 & $96,24 \pm 5,04$ & $95,73 \pm 8,32$ & $95,95 \pm 8,16$ & $96,83 \pm 0,92$ & $96,83 \pm 0,92$ & $96,83 \pm 0,92$ \\
\hline 2 & $96,94 \pm 1,41$ & $96,94 \pm 1,41$ & $96,94 \pm 1,41$ & $96,83 \pm 0,92$ & $96,83 \pm 0,92$ & $96,83 \pm 0,92$ \\
\hline 3 & $96,94 \pm 1,41$ & $96,94 \pm 1,41$ & $96,94 \pm 1,41$ & $96,83 \pm 0,92$ & $96,83 \pm 0,92$ & $96,83 \pm 0,92$ \\
\hline 4 & $96,94 \pm 1,41$ & $96,94 \pm 1,41$ & $96,35 \pm 6,49$ & $96,83 \pm 0,92$ & $96,83 \pm 0,92$ & $96,83 \pm 0,92$ \\
\hline
\end{tabular}

Na base HUG-ILD é possível observar que os melhores resultados foram obtidos na modalidade center, comparando todos os experimentos. Nessa categoria, ocorreram problemas somente quando não houve o aumento de sementes, levando à conclusão de que somente duas sementes, por mais que estejam nos centros dos pulmões, podem não ser suficientes para uma boa segmentação. Na modalidade edge, os problemas continuaram os mesmos, mas o resultado final do coeficiente Dice foi pior que na categoria center. Isso pode indicar que não só faltaram sementes, mas as localização das mesmas prejudicaram a solução do problema.

Já na modalidade random ocorrerem problemas em dois casos, quando não haviam sementes suficientes e quando existiam sementes em excesso. No primeiro caso, o resultado foi pior que em center e melhor que em edge, o que pode sugerir que as localidades das sementes aleatórias eram melhores do que as sementes pertencentes às bordas, mas ainda, em alguns exames, a quantidade de sementes não foi suficiente. Já no segundo caso, com a geração excessiva de sementes, algumas poderiam estar localizadas em regiões fora dos pulmões ou poderiam estar situadas em alguma estrutura interna que não pertencia ao pulmão. Dessa forma, conclui-se que nem sempre uma grande quantidade de sementes implica em uma melhor solução. 
Na base Vessel 12 é possível observar que todas as modalidades em todos os experimentos obtiveram os mesmos resultados de média e desvio padrão do coeficiente Dice. Esse acontecimento pode indicar que, em uma base que contém imagens, em sua maioria, sem ILDs severas, as localidades e quantidades das sementes não fazem tanta diferença quando ocorre uma boa escolha de parâmetros lower e upper, basta somente existir uma semente válida em cada pulmão. Esses resultados mostram a robustez da metodologia pois, ao ser aplicada a diferentes exames, os resultados continuaram iguais.

Embora os experimentos tenham focado em avaliar a influência do posicionamento e quantidade de sementes, é possível observar que, mesmo com uma metodologia simples, os resultados se aproximam dos trabalhos pertencentes ao estado da arte, chegando à 96,94\% $\pm 1,41 \%$ comparado com $97,9 \% \pm 1,0 \%$ [Harrison et al. 2017] e 98,67\% $\pm 0,94 \%$ [Alves et al. 2018] na base HUG-ILD, e 96,83\% $\pm 0,92 \%$ em relação à 99,0\% \pm 0,5\% [Soliman et al. 2017] e 99,19\% $\pm 0,37 \%$ [Alves et al. 2018] na base Vessel 12. Exemplos das segmentações obtidas são apresentados na Figura 6.
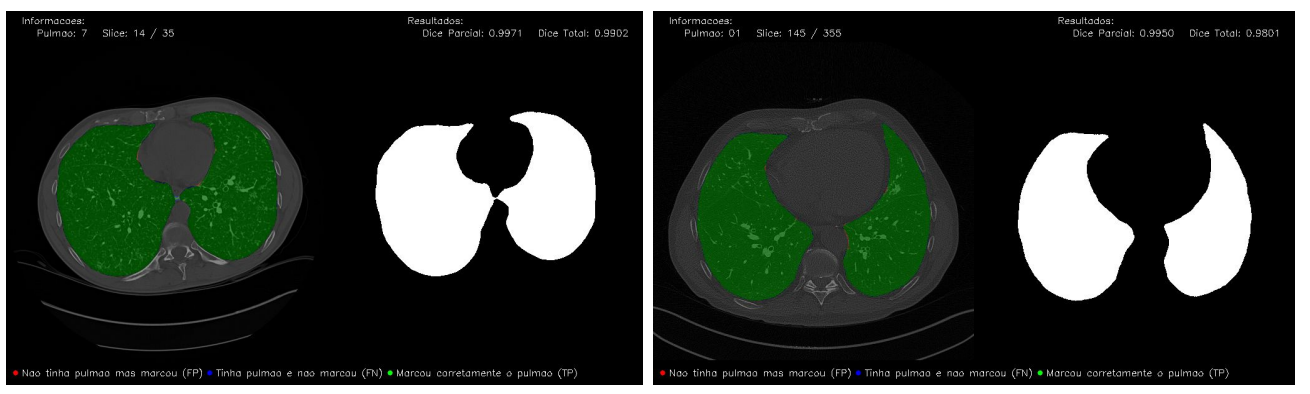

Figura 6. Exemplos dos resultados da segmentação na base HUG-ILD (esquerda) e na Vessel 12 (direita).

\section{Conclusão}

A elaboração desta pesquisa proporcionou uma técnica semi-automática de segmentação de pulmões, incluindo três formas de simular o comportamento de um especialista ao marcar quais áreas das imagens deveriam ser consideradas sementes. Dentre essas formas, a categoria center, que considerou sementes próximas ao centro de cada pulmão, apresentou melhores resultados, já as categorias edge, que visou as bordas, e a random, que gerou sementes em coordenadas aleatórias, alcançaram resultados moderadamente mais baixos, possibilitando a conclusão de que as localidades das sementes impactam ao utilizar o algoritmo de crescimento de região.

Diferentes técnicas foram criadas para melhorar a segmentação inicial obtida com o crescimento de região, como a busca por melhores valores para os parâmetros lower e upper, o aumento de sementes, com categorias que consideravam o volume e as áreas próximas as sementes iniciais, a suavização, que visava corrigir as irregularidades das bordas e preencher os buracos internos dos pulmões, o refinamento, que buscava recuperar os pixels perdidos próximos às bordas, e a separação dos pulmões.

Com experimentos que exploravam a localidade e a quantidade de sementes, considerando casos com escassez e excesso de coordenadas, os melhores resultados chegaram a um coeficiente Dice médio de $96,94 \%$ na base HUG-ILD e de 96,83\% na base Vessel 12, ambos com desvios padrão baixos, $1,41 \%$ e $0,92 \%$ respectivamente. Vale ressaltar que a técnica desenvolvida não precisa ser treinada, portanto não é 
necessária uma elevada quantidade de imagens, bem como um grande poder de processamento. Como trabalho futuro, será feita uma análise da influência do tamanho da imagem na utilização dos filtros morfológicos para verificar se a técnica é invariante à escala ou se sofre alguma degradação quando as imagens são reduzidas.

\section{References}

IARC (2018) "Estimated age-standardized incidence and mortality rates (World) in 2018, worldwide, both sexes, all ages" https://gco.iarc.fr/today/online-analysis-multi-bars. Acessado em: 05/03/2020.

INCA "Tipos de câncer" https://www.inca.gov.br/tipos-de-cancer/cancer-de-pulmao. Acessado em: 05/03/2020.

Thoracic " (2020) Interstitial Lung Disease" https://www.thoracic.org/patients/patient-resources/breathing-in-america/resources/chap ter-10-interstitial-lung-disease.pdf. Acessado em: 05/03/2020.

Vannier, M. W., El-Baz, A., Beache, G. M., Gimel'farb, G., Suzuki, K., Okada, K., Elnakib, A., Soliman, A., Abdollahi, B. (2013) "Computer-Aided Diagnosis Systems for Lung Cancer: Challenges and Methodologies". In: International Journal of Biomedical Imaging, vol. 2013.

Soliman, A., Kthalifa, F., Elnakib, A., El-Ghar, M. A., Dunlap, N., Wang, B., Gimelfarb, G., Keynton, R. and El-Baz, A. (2017) "Accurate lungs segmentation on CT chest images by adaptive appearance-guided shape modeling", In: IEEE transactions on medical imaging, vol. 36, no. 1, p. 263-276.

Harrison, A. P., Xu, Z., George, K., Lu, L., Summers, R. M. and Mollura, D. J. (2017) "Progressive and multipath holistically nested neural networks for pathological lung segmentation from CT images", In: Medical Image Computing and Computer-Assisted Intervention MICCAI 2017: 20th International Conference, Quebec City, QC, Canada, September 11-13, 2017, Proceedings, Part III. Springer International Publishing, 2017, p. 621-629.

Alves, J. H., Neto, P. M. M. and Oliveira, L. F. (2018) "Extracting Lungs from CT Images Using Fully Convolutional Networks”, In: 2018 International Joint Conference on Neural Networks (IJCNN), Rio de Janeiro, p. 1-8.

Depeursinge, A., Vargas, A., Platon, A., Geissbuhler, A., Poletti, P. and Müller, H. (2012) "Building a Reference Multimedia Database for Interstitial Lung Diseases", In: Computerized Medical Imaging and Graphics, vol. 36, no. 3, p. 227-238.

Rudyanto, R. D., Kerkstra, S., van Rikxoort, E. M., Fetita, C., Brillet, P.-Y., Lefevre, C., Xue, W., Zhu, X., Liang, J., Öksüz et al. (2014). "Comparing algorithms for automated vessel segmentation in computed tomography scans of the lung: the VESSEL12 study", In: Medical Image Analysis, vol. 18, no. 7, p. 1217-1232.

Zou, K. H., Warfield, S. K., Bharatha, A., Tempany, C. M., Kaus, M. R., Haker, S. J., Wells, W. M., Jolesz, F. A. and Kikinis, R. (2004) "Statistical validation of image segmentation quality based on a spatial overlap index: Scientific reports", In: Academic radiology, vol. 11, no. 2, p. 178-189. 